Int. J. Morphol.,

24(2):143-146, 2006.

\title{
Modificaciones del Método de Preparaciones Anatómicas de Pulmones Insuflados
}

\author{
Modifications to the Method for Anatomic Preparations of Inflated Lungs \\ Marcelo Gómez Jaramillo \& Pedro Aburto Valdebenito
}

GÓMEZ, J. M. \& ABURTO, V. P. Modificaciones del método de preparaciones anatómicas de pulmones insuflados. Int. J. Morphol., 24(2):143-146, 2006.

RESUMEN: Se describe la técnica y algunas modificaciones al método de preparación de pulmones insuflados para uso en enseñanza morfológica. Esta técnica permite obtener preparaciones óptimas en corto tiempo y adecuadas para su uso en laboratorios de Anatomía Veterinaria.

PALABRAS CLAVE: Pulmones; Preparaciones anatómicas; Anatomía Veterinaria.

\section{INTRODUCCIÓN}

La preparación del pulmones para uso en laboratorios de Anatomía Veterinaria, usualmente reside en la utilización de muestras frescas in situ, conservadas en formaldehído u otras soluciones preservantes (McCulloh \& Rutty 1998), microdisección del árbol bronquial (Ishaq, 1980; Plopper et al., 1983), técnicas de corrosión (Viggiano et al., 2003) o inyecciones con látex del árbol bronquial (Nettum, 1993). Nuevas técnicas anatómicas como la plastinación, que utilizan polímeros que se incorporan a los espacios intracelulares y extracelulares de los tejidos, también han ofrecido preparaciones adecuadas para estudio de los pulmones (Henry, 1987, 1992a y 1992b). No obstante, esta técnica todavía es cara de implementar y requiere largos tiempos de impregnación forzada al vacío del polímero en el tejido pulmonar.

Preparaciones que representen adecuadamente los pulmones inflados son necesarias para entender la disposición, anatomía y fisiología de estos órganos respiratorios. El presente trabajo describe algunas modificaciones del método descrito por McKiernan \& Kneller (1983) para preparar muestras de pulmones insuflados de animales domésticos. Si bien esta técnica es utilizada en muchos Departamentos de Anatomía Veterinaria a nivel mundial, existen muy pocas publicaciones referidas a su metodología, complicaciones y perfeccionamientos.

A continuación, se describe la técnica de insuflado incluyendo modificaciones para una mejor preparación de las muestras, un rápido proceso y una mejor conservación.

\section{TÉCNICA}

Para una preparación adecuada de pulmones insuflados se requiere que éstos sean extraídos inmediatamente después de sacrificar el animal. Es deseable, previa heparinización del animal (perro y gato: $6.000 \mathrm{UI}$; bovinos y equinos: 25.000 UI) para evitar la formación de coágulos y facilitar un mejor vaciado de los vasos pulmonares. La extracción de los pulmones se efectúa disecando una ventana torácica realizada mediante secciones a nivel de la unión costocondral y cerca de la unión costovertebral. No es conveniente el uso de muestras fijadas o pulmones dañados (con laceraciones o contusiones) por patologías respiratorias, o bien, por lesiones iatrogénicas durante la extracción de los especímenes. Esto debido a que lesiones en el parénquima pulmonar pueden producir una incompleta distensión de los lóbulos pulmonares durante el proceso posterior de secado e insuflado. Se debe, por tanto, tener especial cuidado durante la extracción y disección de la tráquea y los pulmones.

La disección debe incluir la remoción de los tejidos conectivo y graso, venas pulmonares, arterias pulmonares, corazón (si fue extraído con los pulmones), coágulos y nódulos linfáticos asociados. Este trabajo de disección previa permite un secado más rápido de los pulmones y una mejor presentación de la preparación final. Posteriormente, se realiza el lavado externo e interno de los pulmones. Este lavado o "flushing" puede efectuarse con agua corriente a presión moderada. Nosotros combrobamos que el uso de 
agua destilada más la administración de agua oxigenada al $5 \%$ produce mejores resultados, al conferir un color más claro y homogéneo a los pulmones. El lavado se realiza mediante la inyección o administración directa de agua destilada, o su equivalente, a través de la tráquea. Junto con ello, se realiza el masaje digital de los lóbulos pulmonares, para así producir un mejor drenaje del árbol bronquial y facilitar, además, una distensión homogénea de los lóbulos pulmonares. Luego se invierte el pulmón y se realiza la compresión manual y en dirección dorsoventral de los lóbulos pulmonares en forma delicada con el fin de producir el vaciado del agua mezclada con sangre. Este procedimiento debe repetirse hasta observar desde la tráquea la aparición de un fluido claro y no sanguinolento. Después del lavado minucioso los pulmones adquieren una coloración más blanquecina.

Luego se procede a adecuar un tubo de goma o plástico al interior de la tráquea. Tubos con diámetro de 5-7 $\mathrm{mm}$ son adecuados para especímenes felinos y caninos pequeños y de 13-15 mm para especímenes caninos medianos y grandes y pequeños rumiantes. Este tubo es fijado a la tráquea mediante el uso de gasas, cintas adhesivas y/o elástico, o bien, mediante una abrazadera metálica y pin- zas hemostáticas (Fig. 1). De esta forma se sostienen la tráquea y ambos pulmones en forma vertical. El tubo es luego conectado a un compresor de aire u otra fuente de aire comprimido. En nuestro laboratorio utilizamos un compresor portátil de $1.5 \mathrm{Hp}$ y $1.1 \mathrm{Kw}$ (ABAC air compressor, Tecnofer S. A.) para preparaciones de especímenes caninos, felinos y pequeños rumiantes. Compresores de mayor poder (> 1.5 Hp) son necesarios para preparaciones de especímenes bovinos y equinos. Inicialmente, los pulmones son inflados mediante apertura de la fuente de aire comprimido en forma lenta. Durante el inflado de los lóbulos pulmonares se procede nuevamente al masaje de ellos para facilitar así su distensión. Esto permite una diferenciación clara de los lóbulos pulmonares y las fisuras interlobares. Durante el inflado se produce drenaje del líquido remanente a través del hilio pulmonar.

Una vez que los pulmones han adquirido una forma aceptable (Fig. 1B), es conveniente fijar la presión de inyección de aire a ese nivel. Para pulmones de perros medianos (15 kg aprox.), presiones de 110 psi (7 barr) resultan apropiadas para lograr un insuflado adecuado de las preparaciones (Fig. 2). Es necesario observar cuidadosamente el nivel de presión ya que una presión excesiva pue-
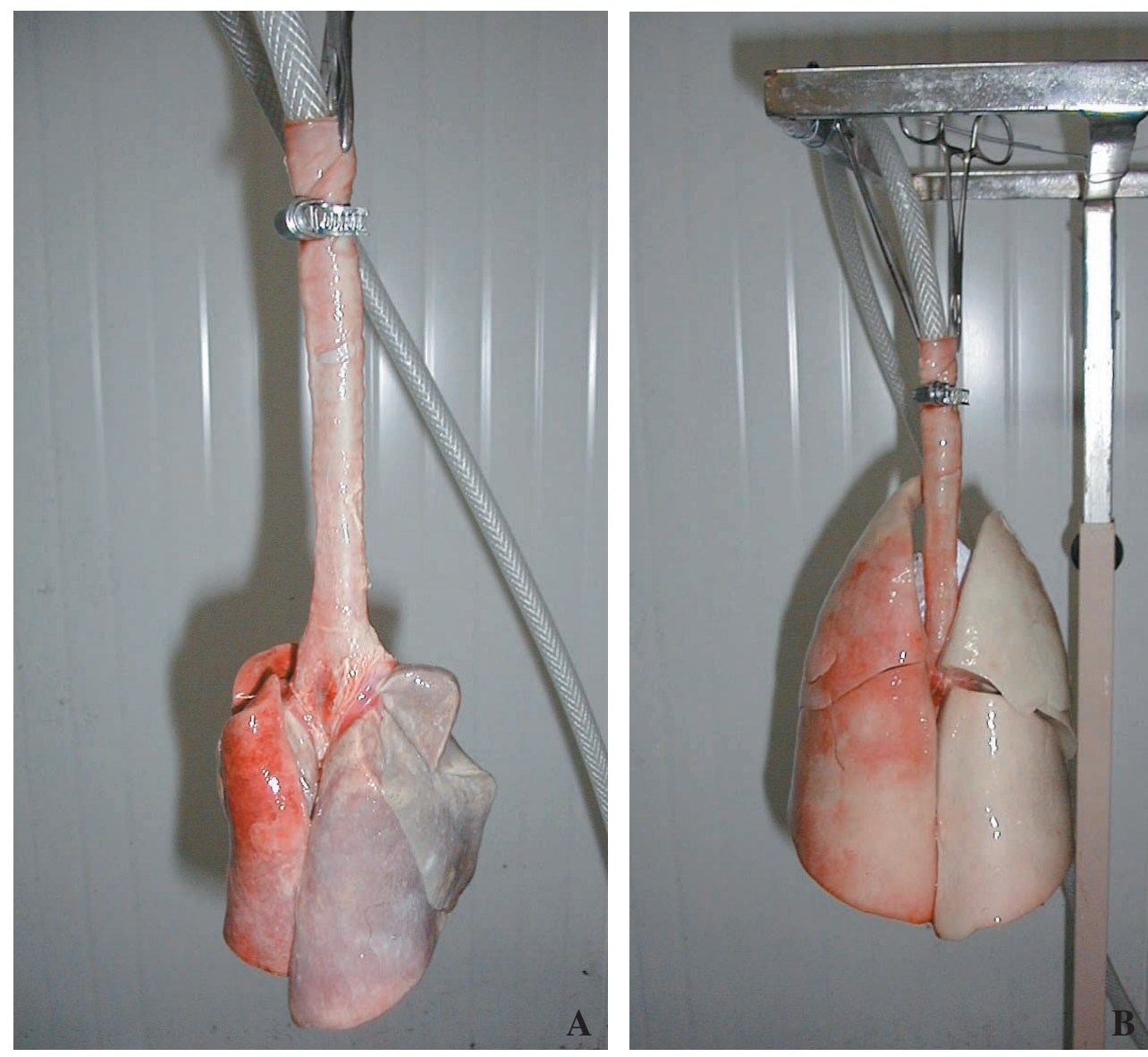

Fig. 1. Disposición y suspensión de pulmones de perro para el insuflado (vista dorsal). A. Previo al inflado; observe sujeción con abrazadera metálica y pinzas hemostáticas. B. Luego del insuflado completo; observe la conexión hacia la fuente de aire comprimido a través de la tráquea. Se observa congestión hipostática del pulmón derecho. 

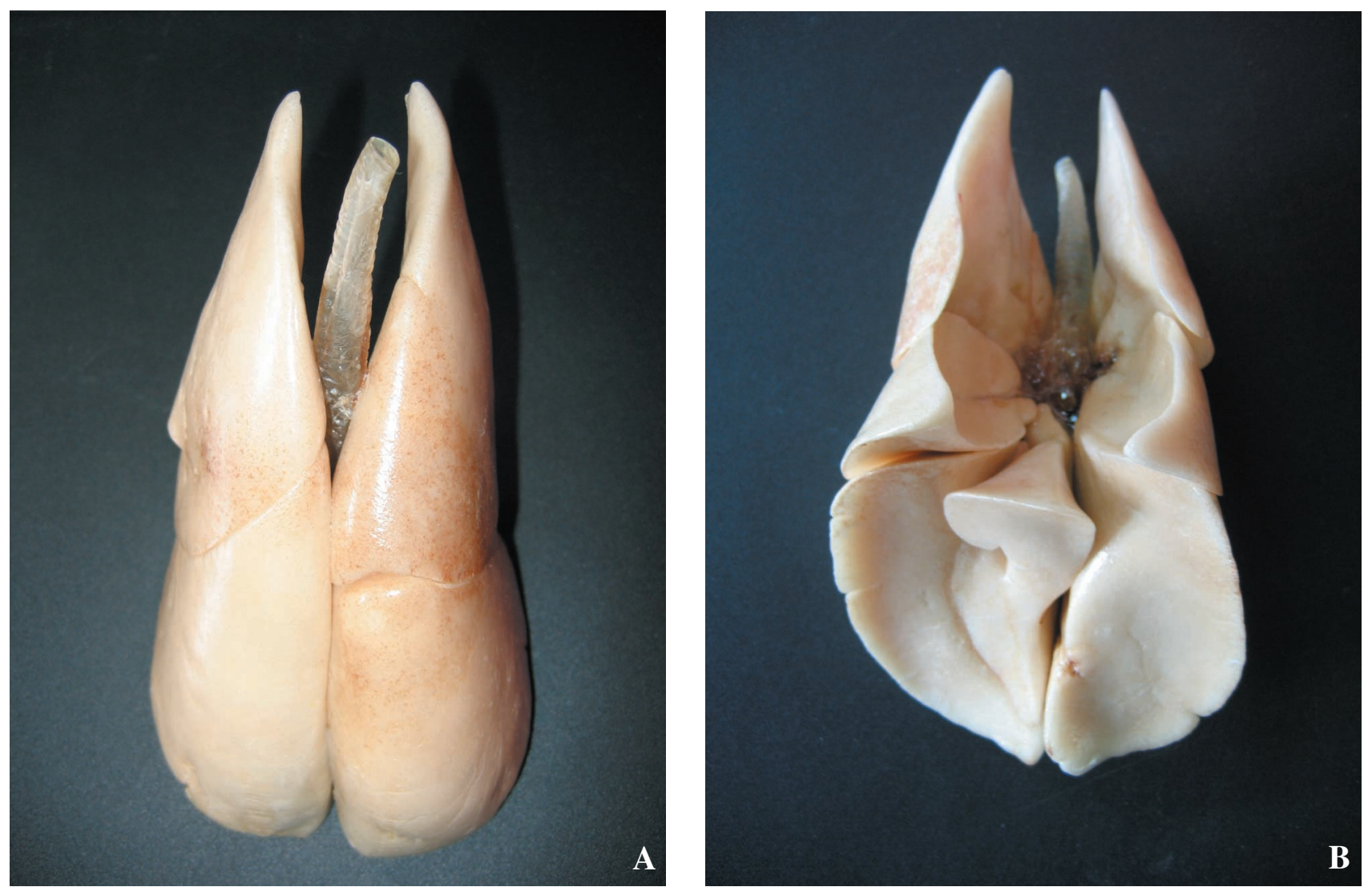

Fig. 2. Preparación final de insuflado de pulmones de gato. A. Vista dorsal B. Vista ventral. Observe en ambas vistas la disposición clara de los lóbulos pulmonares y las fisuras interlobares.

de producir ruptura del parénquima y pleura visceral pulmonar, observándose zonas de elevación semejantes a enfisemas o bien se produce la explosión de la muestra. En la superficie medial de los pulmones, a nivel de las impresiones cardíacas derecha e izquierda, es posible adicionar papel absorbente enrollado para moldear el tamaño del corazón y además facilitar al secado del especimen. El tiempo de insuflado es variable para las distintas especies. McKiernan y Kneller indican que períodos de 3 a 4 días de insuflado son necesarias para pulmones de gatos y alrededor de 7 días para insuflados de perro. Para especímenes mayores, como equinos o bovinos, estos autores establecen tiempos de hasta 2 semanas. Por otra parte, hemos encontrado que adicionando una fuente de aire externa (ventilador) es posible reducir hasta la mitad el tiempo de prepara- ción. Sin embargo, tiempos menores no son recomendados ya que producirán colapso y/o arrugamiento del la pleura visceral pulmonar. Es necesaria una buena conservación posterior de las preparaciones. Para ello, es conveniente dejar reposar las piezas por un período de 2 semanas y luego conservarlas en bolsas de plástico con cierre de sello (ej. Ziploc), para evitar infestación de insectos y daño mecánico de las piezas. Para el mismo propósito, se recomiendan colocar pequeñas cantidades de cristales de naftaleno al interior del árbol bronquial. Adicionalmente, y con el objeto de impermeabilizar las preparaciones y evitar su daño por la humedad y polvo, en nuestro laboratorio se aplica laca acrílica transparente en spray sobre las preparaciones insufladas, lo que además otorga un aspecto brillante a los especimenes confiriéndoles una apariencia más natural.

GÓMEZ, J. M. \& ABURTO, V. P. Modifications to the method for anatomic preparations of inflated lungs. Int. J. Morphol., 24(2):143146, 2006.

SUMMARY: The technique and modifications of preparation of air-dried inflated lung specimens is presented. This method produces lung specimens in a short period and adequate for teaching in Veterinary Anatomy labs.

KEY WORDS: Lungs; Anatomical preparations; Veterinary Anatomy. 


\section{REFERENCIAS BIBLIOGRÁFICAS}

Henry, R. Plastination of an integral heart-lung specimen. $J$. Int. Soc. Plastination, 1:20-2, 1987.

Henry, R. Silicone tracheobronchial casts. J. Int. Soc. Plastination, 6:29-33, 1992a.

Henry, R. Silicone pulmonary vascular casts with attached tracheobronichial cast. J. Int. Soc. Plastination., 6:41-4, $1992 b$.

Ishaq, M. A morphological study of the lungs and bronchial tree of the dog: with a suggested system of nomenclature for bronchi. J. Anat, 131:589-610, 1980.

McCulloch, T. A. \& Rutty, G. N. Postmortem examination of the lungs: a preservation technique for opening the bronchi and pulmonary arteries individually without transection problems. J. Clin. Pathol., 51:163-4, 1998.

McKiernan, B. \& Kneller, S. A simple method for the preparation of inflated air-dried lung specimens. Vet. Radiol., 24:58-62, 1983.

Nettum, J. Bronchoalveolar casting using formalin-fixed canine lungs and low viscosity silicone rubber. Anat. Rec., 236:408-10, 1993.

Plopper, C. G.; Mariassy, A. T. \& Lollini, L. O. Structure as revealed by airway dissection. A comparison of mammalian lungs. Am. Rev. Respir. Dis., 128: S4-7, 1983.

Viggiano D.; Sangiorgi S.; Reguzzoni M.; Manelli A.; Marano L.; Dell'Orbo C \& Passiatore C. A new method to make vascular and bronchial casts of voluminous organs. Eur. J. Morphol. 41:161-5, 2003.
Dirección para correspondencia: Dr. Marcelo Gómez Jaramillo Instituto de Anatomía Veterinaria Universidad Austral de Chile Campus Isla Teja, Casilla 567 Valdivia - CHILE

Fono 56-63-221071

Fax 56-63-221473

Email:marcelogomez@uach.cl

Recibido : 18-01-2006

Aceptado: 07-03-2006 